\title{
EFFUSIVE-CONSTRICTIVE PERICARDITIS POST SURGICAL REVISION FOR IATROGENIC HEMOPERICARDIUM
}

\author{
Martin Kolek
}

\author{
Department of Cardiac Surgery, University Hospital Ostrava, Czech Republic \\ E-mail: martin.kolek@fno.cz
}

Received: October 28, 2010; Accepted: December 1, 2010

Key words: Percutaneous coronary intervention/Hemopericardium/Pericarditis/Pericardiocentesis/Pericardiectomy

\begin{abstract}
Aim. A case report of subacute effusive-constrictive pericarditis in a patient with iatrogenic hemopericardium is presented.

Methods. A 69-year-old man was referred to our department for percutaneous coronary intervention complicated with hemopericardium with cardiac tamponade. Continuous bleeding after pericardiocentesis required an urgent surgical revision with evacuation of hemopericardium and local treatment of the sources of bleeding. A mild to moderate pericardial effusion persisted in the post-operative period, without any symptoms of cardiac tamponade. A global heart failure developed in the patient eleven months after complicated coronary intervention (surgical revision). A technically successful pericardiocentesis did not improve the clinical state of the patient. Echocardiography and magnetic resonance imaging confirmed the diagnosis of effusive-constrictive pericarditis.

Results. Subsequent pericardiectomy resulted in a prompt and complete relief of symptoms and signs of heart failure.

Conclusion. Effusive-constrictive pericarditis is an uncommon disorder characterised by symptoms of refractory cardiac failure, thickening of the visceral pericardium and pericardial effusion, with no improvement after pericardiocentesis. In indicated cases, pericadiectomy leads to recovery in a large percentage of patients.
\end{abstract}

\section{INTRODUCTION}

Effusive-constrictive pericarditis is an uncommon disorder characterised by thickening of the visceral pericardium and the presence of tense pericardial effusion. The syndrome can occur in various types of pericarditis with effusion but is relatively more frequent as a result of tuberculosis, neoplasia or after chest radiation. Cardiac surgery is a rare cause of effusive-constrictive pericarditis. In our case, the onset of the disorder was related to hemopericardium, which was a primary complication of a percutaneous coronary intervention (PCI) and which persisted in the form of haemorrhagic pericardial effusion even after urgent surgical revision. The options for diagnostics and therapy of effusive-constrictive pericarditis are discussed.

\section{CASE REPORT}

We present a case report of effusive-constrictive pericarditis in a 69-year-old man. He was a polymorbid patient with the history of hypertension, diabetes mellitus, dyslipidaemia, bronchial asthma, repeated vertebrobasilar transient ischemic attack, ischemic heart disease, myocardial infarction of unspecified site and paroxysmal atrial fibrillation.

The patient presented with unstable angina pectoris and therefore he underwent PCI for severe stenosis of the left anterior descending (LAD) artery with a stent implantation. After 10 days another coronary angiography was carried out due to recurrent angina pectoris. A PCI of right coronary artery (RCA) with implantation of three drug-eluting stents followed. The procedure was complicated with iatrogenic perforation of RCA, accompanied by hemopericardium and cardiac tamponade. The condition was resolved with prolonged inflation of angioplasty balloon in the area of perforation and subsequent pericardiocentesis. The volume of blood aspirated reached $1400 \mathrm{ml}$. Due to the continuous bleeding, urgent surgical revision was performed. The main source of bleeding was not the RCA but an injury to the heart in the area of the left marginal artery (haematoma) - incurred during pericardiocentesis. Furthermore, a hemoperitoneum was diagnosed, manifested by infiltration of blood at the diaphragm in the median line (also a complication of the pericardiocentesis). The surgical procedure consisted of aspiration of blood and blood clots from the pericardium $(200 \mathrm{ml})$ and from the peritoneal cavity, local treatment of the sources of bleeding, and drainage.

Despite the hypotension during the preoperative period, we observed no neurological deficit after surgery. The drop in blood count was saturated with blood transfers; we also adjusted the hypocoagulation caused by heparin and dual antiaggregation treatment. The circulatory parameters were stable; the paroxysm of atrial fibrillation was converted pharmacologically (amiodaron) to sinus rhythm. A repeated pleurocentesis to evacuate the effusion was carried out during hospitalisation. The patient was discharged after three weeks, in a good medical state, with a small residual pericardial effusion $(6-10 \mathrm{~mm}$ along 


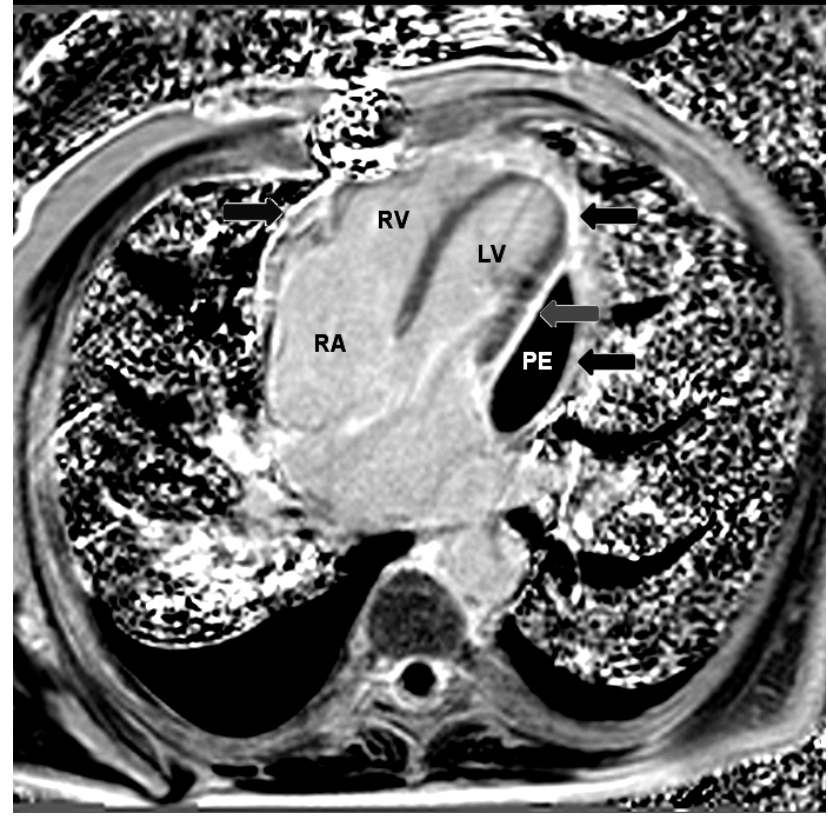

Fig. 1. Magnetic resonance, four-chamber view, pericardial effusion along the lateral wall of the left ventricle, pericardial thickening (arrows) and the presence of a bilateral, especially right-sided pleural effusion.

$\mathrm{LV}=$ left ventricle, $\mathrm{PE}=$ pericardial effusion, $\mathrm{RA}=$ right atrium, $\mathrm{RV}=$ right ventricle

the right ventricle, inferior, posterior and lateral wall of the left ventricle).

In the course of out-patient follow-up, a small to medium pericardial effusion persisted, with no signs of cardiac tamponade.

Eleven months after the complicated PCI, the patient was hospitalised for global heart failure - progressive dyspnea, lower limb oedema, borderline liver size and filling level of jugular veins, bilateral pleural effusion, small ascites and dyspepsia. Transthoracic echocardiography demonstrated a moderate-sized loculated pericardial effusion. The effusion was distributed along the right ventricle and behind the basis of lateral and dorsal left ventricle wall, together with echogenous pericardium. The left ventricle systolic function was good (ejection fraction $55 \%$ ). A slightly abnormal septal motion was observed. Restrictive left ventricular filling pattern was assessed by Doppler echocardiography; the diastolic mitral inflow varied by $20 \%$ of the peak E-wave velocity. There were mild mitral and tricuspid regurgitations. The diameter of the inferior vena cava was again at the borderline; its inspiratory collapse was minimal.

We continued the treatment with pericardiocentesis, accompanied by a single aspiration of $100 \mathrm{ml}$ of haemorrhagic effusion, with minimal residuum behind the left ventricular posterior wall. However, severe symptoms of heart failure persisted, with an early recurrence of the effusion. Subsequently magnetic resonance imaging (MRI) was performed which revealed, apart from a moderatesized pericardial effusion, also a thickening of the peri-

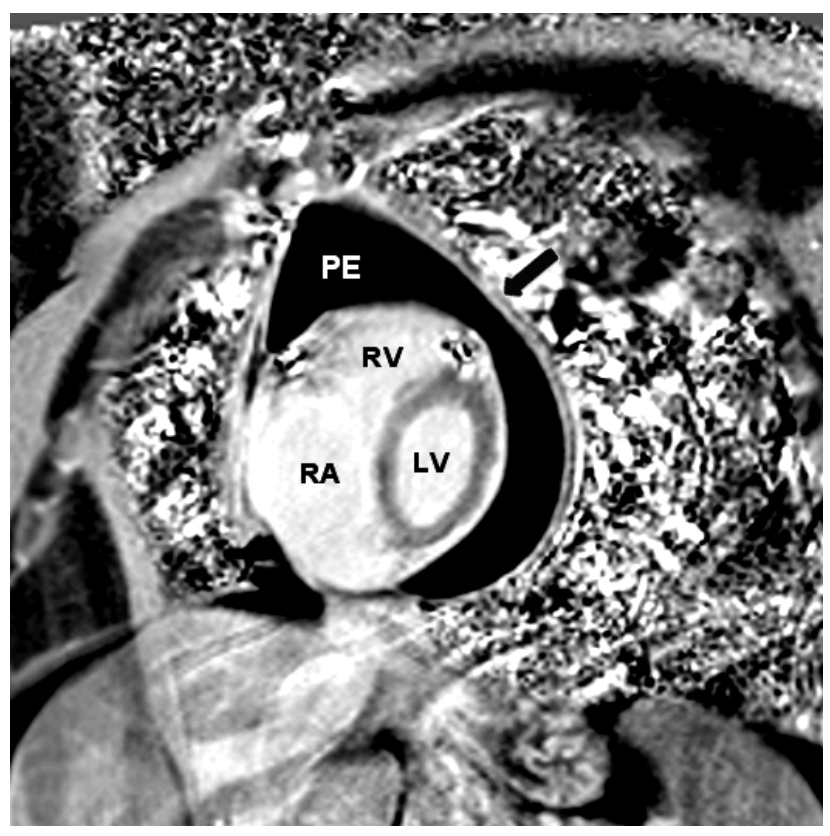

Fig. 2. Magnetic resonance, modified short-axis view, pericardial effusion along the right and left ventricle, thickened pericardium (arrow). $\mathrm{LV}=$ left ventricle, $\mathrm{PE}=$ pericardial effusion, $\mathrm{RA}=$ right atrium, $\mathrm{RV}=$ right ventricle

cardium up to 6-7 $\mathrm{mm}$ (Fig. 1 and 2 ). The finding was diagnosed as effusive-constrictive pericarditis.

The patient underwent pericardiectomy with aspiration of the effusion. The surgeon found fibrotic thickening of both visceral and parietal pericardium. The surgical procedure consisted of wide removal of the parietal and visceral pericardium. Histopathological examination of the resected pericardium showed fibrotic thickening and a foreign body granuloma. No cancer cells were detected upon cytology examination of the pericardial effusion; the cultures for detection of bacteria were negative (incl. tuberculous mycobacterium).

The clinical manifestations of cardiac failure receded immediately after the surgery. In the post-operative period, the patient suffered from acute exacerbation of chronic bronchitis. Pleurocentesis to evacuate the effusion was carried out bilaterally. The patient was stable in the course of two-year outpatient follow-up, with no signs of heart failure.

\section{DISCUSSION}

Effusive-constrictive pericarditis is an uncommon disorder characterised by the presence of pericardial effusion and constriction of the heart caused by the visceral pericardium. The syndrome was described in the literature several decades ago by Burchell and Spodick ${ }^{1,2}$. The knowledge related to effusive-constrictive pericarditis results from case reports and clinical trials with a limited 
number of participants only ${ }^{3-9}$. In the prospective study carried out by Sagristà-Sauleda ${ }^{7}$, the prevalence of this disorder was $1.3 \%$ in patients with pericardial disease of any type and $6.9 \%$ in patients with cardiac tamponade.

The classical rigid form of constrictive pericarditis is characterised by limitation in the diastolic filling of all heart chambers. An elevation and equilibration of atrial and ventricular early diastolic pressures are observed. Diastolic ventricular pressures show a dip-and-plateau morphology. The curve of venous pressure and pulse is "M" or "W" shaped (deep descent "y", as well as deep systolic descent " $\mathrm{x}$ ”). During respiration, the changes in intrathoracic pressure are not transferred into the pericardial space and heart chambers.

In subacute elastic form of constrictive pericarditis, the filling of the heart is limited with thickened elastic pericardium for the whole period of the heart cycle. The changes of intrathoracic pressure are transmitted into the heart chambers during respiration. The pressure curves resemble more a condition of heart tamponade rather than rigid constrictive pericarditis. The diastolic descent " $y$ " of the jugular venous pressure curve is missing during a heart tamponade.

For the effusive-constrictive pericarditis it is typical, that after pericardiocentesis, the intrapericardial pressure drops to $0 \mathrm{~mm} \mathrm{Hg}$ or may be slightly negative, however the elevated right atrial pressure persists, as well as a dip-plateau morphology of right ventricular pressure.

To make an accurate diagnosis of effusive-constrictive pericarditis can be difficult. It is therefore necessary to bear this condition in mind in the course of differential diagnostics in patients with a significant pericardial effusion of various ethiologies and clinical manifestation of a cardiac tamponade, especially if the symptoms and signs of the tamponade persist even after removal of pericardial fluid.

Echocardiography is one of the most important examinations in patients with cardiac constriction and tamponade. The hallmarks of constrictive pericarditis are: thickened echogenous pericardium, abnormal ventricular septal motion (the septum moves to the left during inspirium, to the right during expirium), the inferior vena cava is dilated with lack of inspiratory collapse ${ }^{10}$. The diastolic left ventricular function is abnormal (restrictive filling pattern at Doppler echocardiography). The diagnosis of cardiac tamponade is based on the following echocardiographic features: the presence of pericardial effusion, diastolic collapse of the right atrium and ventricle, in serious cases also collapse of the left ventricle, dilated inferior vena cava, noncollapsing at inspiration (< $35 \%$ of its diameter $)^{11}$, increased respiratory variability of diastolic mitral and tricuspid or systolic aortic flow velocities - significant decrease in mitral and aortic valve flow (> 25\%) and increased flow through tricuspid orifice with inspiration (less accurate). Effusive-constrictive pericarditis is characterized by mixed findings.

Examination of the heart with computed tomography (CT) and magnetic resonance (MRI) is also very informative $^{12}$. CT is a more sensitive technique than echocardiography for evaluation of pericardial thickness and very precisely depicts the pericardial calcifications. MRI presents an ideal diagnostic method for the assessment of morphological and functional parameters related to the pericardial diseases ${ }^{13}$. We may obtain precise information about the thickness of the pericardium, presence, size and localisation of the pericardial effusion. MRI can detect a limited filling of the heart ventricles during the early diastole as well as abnormal motion of the ventricular septum caused by changes in trans-septal gradient during respiration. Pericardial thickening greater than $4 \mathrm{~mm}$ and a characteristic hemodynamic finding help us to differentiate between constrictive pericarditis and restrictive cardiomyopathy.

It is possible to confirm the diagnosis of effusiveconstrictive pericarditis (as mentioned above) with simultaneous registration of intrapericardial pressure and the pressure curve from the right atrium and ventricle during pericardiocentesis. Constrictive hemodynamics persists after evacuation of the effusion.

A spontaneous resolution of constriction after pericardiocentesis is possible, especially in patients with idiopatic exudative acute pericarditis (transient cardiac constriction $)^{7,14-16}$. However, a persistence of severe symptoms and signs of heart failure and of hemodynamic abnormalities is an indication for pericardiectomy, which is followed by prompt improvement in most patients. The symptomatic improvement is limited only in patients referred to surgery in advanced stage of constrictive pericarditis. This is mainly due to the fact that it is not possible to completely remove the constriction, or may be caused by fibrosis and atrophy of the myocardium. The operative mortality of pericardiectomy is about $5-6 \%$ (ref. $^{17,18}$ ).

In this report, we describe a case of subacute effusiveconstrictive pericarditis in a patient after PCI, complicated with hemopericardium and cardiac tamponade, followed by an urgent surgical revision and persisting haemorrhagic pericardial effusion. The diagnosis was established upon the clinical findings (no improvement in clinical status after pericardiocentesis) and with the use of imaging methods (echocardiography, MRI). We did not carry out a right heart catheterization due to the unequivocal clinical finding. Subtotal pericardiectomy as a treatment method of first choice, resulted in a complete adjustment of the patient's medical state.

\section{CONCLUSION}

Effusive-constrictive pericarditis presents a rare nosologic unit. It typically manifests as persisting symptoms and signs of heart failure, despite successful pericardiocentesis. Various imaging methods aid correct diagnostics - echocardiography, computed tomography and magnetic resonance imaging. However, simultaneous registration of intrapericardial pressure and the pressure curve from the right atrium and ventricle during pericardiocentesis is the gold standard. The establishment of correct diagnosis is very important, as the surgical intervention (pericardiectomy) leads to a complete recovery in a large percentage of patients. 


\section{REFERENCES}

1. Burchell HB. Problems in the recognition and treatment of pericarditis. J Lancet 1954;74:465-470.

2. Spodick DH, Kumar S. Subacute constrictive pericarditis with cardiac tamponade. Dis Chest 1968;54:62-66.

3. Cameron J, Oesterle SN, Baldwin JC, Hancock EW. The etiologic spectrum of constrictive pericarditis. Am Heart J 1987;113:354360.

4. Russell JB, Syed FF, Ntsekhe M, Mayosi BM, Moosa S, Tshifularo M, Smedema JP. Tuberculous effusive-constrictive pericarditis. Cardiovasc J Afr 2008;19:200-201.

5. Zagol B, Minderman D, Munir A, D'Cruz I. Effusive constric tive pericarditis: 2D, 3D echocardiography and MRI imaging. Echocardiography 2007;24:1110-1114.

6. Ito M, Tanabe Y, Suzuki K, Kumakura M, Nakayama K, Kanazawa H, Yamazaki Y, Aizawa Y. A case of effusive-constrictive pericarditis after cardiac surgery. Mayo Clin Proc 2001;76:555-558.

7. Sagristà-Sauleda J, Angel J, Sánchez A, Permanyer-Miralda G, Soler-Soler J. Effusive-constrictive pericarditis. N Engl J Med 2004;350:469-475.

8. Hancock EW. Subacute effusive-constrictive pericarditis. Circulation 1971;43:183-192.

9. Nakayama Y, Ohtani Y, Kobayakawa N, Kobayashi J, Kaneko Y Aoyagi T. A case of early phase dialysis associated effusive constrictive pericarditis with distinct surgical findings. Int Heart $\mathrm{J}$ 2009;50:685-691.

10. Himelman RB, Lee E, Schiller NB. Septal bounce, vena cava plethora, and pericardial adhesion: informative two-dimensional echocardiographic signs in the diagnosis of pericardial constriction. J Am Soc Echocardiogr 1988;1:333-340.

11. Himelman RB, Kircher B, Rockey DC, Schiller NB. Inferior vena cava plethora with blunted respiratory response: a sensitive echocardiographic sign of cardiac tamponade. J Am Coll Cardiol 1988;12:1470-1477.

12. Breen JF. Imaging of the pericardium. J Thorac Imaging 2001;16:47-54

13. Frank H, Globits S. Magnetic resonance imaging evaluation of myocardial and pericardial disease. J Magn Reson Imaging 1999; 10:617-626.

14. Woods T, Vidarsson B, Mosher D, Stein JH. Transient effusiveconstrictive pericarditis due to chemotherapy. Clin Cardiol 1999;22:316-318

15. Sagristà-Sauleda J, Permanyer-Miralda G, Candell-Riera J, Angel J, Soler-Soler J. Transient cardiac constriction: an unrecognized pattern of evolution in effusive acute idiopathic pericarditis. Am J Cardiol 1987;59:961-966.

16. Tanaka K, Kawauchi M, Murota Y, Takamoto I, Ikenouchi H, Hada Y, Furuse A. Reversible subacute effusive-constrictive pericarditis after correction of double-chambered right ventricle: a case report. J Cardiol 2002;39:267-270.

17. Clare GC, Troughton RW. Management of constrictive pericarditis in the 21st century. Current treatment options in cardiovascular medicine 2007;9:436-442

18. Schwefer M, Aschenbach R, Heidemann J, Mey C, Lapp H Constrictive pericarditis, still a diagnostic challenge: comprehensive review of clinical management. Eur J Cardiothorac Surg 2009;36:502-510. 